\title{
Water-separated ion pairs cause the slow dielectric mode of magnesium sulfate solutions
}

Cite as: J. Chem. Phys. 148, 222812 (2018); https://doi.org/10.1063/1.5000385

Submitted: 15 August 2017 . Accepted: 23 December 2017 . Published Online: 28 February 2018

Shavkat I. Mamatkulov, Klaus F. Rinne, Richard Buchner (D), Roland R. Netz (D), and Douwe Jan Bonthuis

\section{ARTICLES YOU MAY BE INTERESTED IN}

Calcium ions in aqueous solutions: Accurate force field description aided by ab initio molecular dynamics and neutron scattering

The Journal of Chemical Physics 148, 222813 (2018); https://doi.org/10.1063/1.5006779

The influence of polarizability and charge transfer on specific ion effects in the dynamics of aqueous salt solutions

The Journal of Chemical Physics 148, 222803 (2018); https://doi.org/10.1063/1.5012682

A relationship between the effect of uni-univalent electrolytes on the structure of water and on its volatility

The Journal of Chemical Physics 148, 222807 (2018); https://doi.org/10.1063/1.5009311

Where in the world is AIP Publishing?

Find out where we are exhibiting next 


\title{
Water-separated ion pairs cause the slow dielectric mode of magnesium sulfate solutions
}

\author{
Shavkat I. Mamatkulov, ${ }^{1,2}$ Klaus F. Rinne, ${ }^{1}$ Richard Buchner, ${ }^{3}$ Roland R. Netz, ${ }^{1}$ \\ and Douwe Jan Bonthuis ${ }^{1, a)}$ \\ ${ }^{1}$ Fachbereich Physik, Freie Universität Berlin, 14195 Berlin, Germany \\ ${ }^{2}$ Ion-Plasma and Laser Technologies Institute of the Uzbekistan AS, Tashkent, Uzbekistan \\ ${ }^{3}$ Institut für Physikalische und Theoretische Chemie, Universität Regensburg, D-93040 Regensburg, Germany
}

(Received 15 August 2017; accepted 23 December 2017; published online 28 February 2018)

\begin{abstract}
We compare the dielectric spectra of aqueous $\mathrm{MgSO}_{4}$ and $\mathrm{Na}_{2} \mathrm{SO}_{4}$ solutions calculated from classical molecular dynamics simulations with experimental data, using an optimized thermodynamically consistent sulfate force field. Both the concentration-dependent shift of the static dielectric constant and the spectral shape match the experimental results very well for $\mathrm{Na}_{2} \mathrm{SO}_{4}$ solutions. For $\mathrm{MgSO}_{4}$ solutions, the simulations qualitatively reproduce the experimental observation of a slow mode, the origin of which we trace back to the ion-pair relaxation contribution via spectral decomposition. The radial distribution functions show that $\mathrm{Mg}^{2+}$ and $\mathrm{SO}_{4}^{2-}$ ions form extensive water-separated-and thus strongly dipolar-ion pairs, the orientational relaxation of which provides a simple physical explanation for the prominent slow dielectric mode in $\mathrm{MgSO}_{4}$ solutions. Remarkably, the $\mathrm{Mg}^{2+}-\mathrm{SO}_{4}^{2-}$ ion-pair relaxation extends all the way into the $\mathrm{THz}$ range, which we rationalize by the vibrational relaxation of tightly bound water-separated ion pairs. Thus, the relaxation of divalent ion pairs can give rise to widely separated orientational and vibrational spectroscopic features. Published by AIP Publishing. https://doi.org/10.1063/1.5000385
\end{abstract}

\section{INTRODUCTION}

Sulfate ions are important in physiology and biochemistry. ${ }^{1,2}$ For example, addition of ammonium sulfate in high concentration reduces the solubility of proteins in aqueous solution ${ }^{3}$ and is commonly used for protein purification. ${ }^{4}$ The general mechanisms by which ions affect the solvation water structure and thereby the solubility of other substances have been the subject of intense debate. ${ }^{5-9}$ Solutions of $\mathrm{MgSO}_{4}$ have been shown to be particularly interesting, since dielectric relaxation studies point to pronounced ion-pairing effects with a clear spectroscopic signature in the sub-GHz range, in addition to the water relaxation peak, which is present at a frequency of $f \approx 20 \mathrm{GHz} \cdot{ }^{10}$ Moreover, THz absorption and fs infrared spectra have been interpreted in terms of significant deceleration of the hydration water dynamics, which has been found to depend nonadditively on the combination of anions and cations, ${ }^{11}$ suggesting an effect of high-frequency correlations between the anion, the cation, and the hydration water. The conventional framework for interpreting experimental dielectric spectra is based on Debye relaxation, which in its simplest form involves a single dipole relaxation time. Typically, molecular dynamics (MD) simulations do not yield dielectric spectra but are compared with an interpretation of experimental spectra extracted from multiple Debye fits instead. ${ }^{12-16}$ This approach, however, relies on the assumptions that the individual spectral contributions

a)Electronic mail: d.j.bonthuis@fu-berlin.de are of Debye form and that the total spectrum follows from a simple superposition of single Debye processes. Simulations suggest that both these assumptions are incorrect. ${ }^{17}$ Moreover, it is common to attribute the Debye contribution with the longest relaxation time to the formation of dipolar ion pairs. ${ }^{18}$ Without careful analysis, this assignment is premature. For example, $\mathrm{NaCl}$ solutions show more than a single Debye relaxation peak, ${ }^{19}$ but the contribution from ion pairing is negligible. ${ }^{17}$

An important advance in the interpretation of experimental electrolyte dielectric spectra is provided by the direct comparison with spectra derived from molecular simulations, because the partitioning into ionic and water contributions, as well as the further decomposition into contributions from different solvation shells, is only possible in simulated spectra. ${ }^{17,20-22}$ A quantitative comparison with experiments can be based on heuristic fits of the Cole-Cole or Havriliak-Negami equations, for example. ${ }^{23,24}$ Presently, the biggest drawback of classical molecular dynamics simulations of dielectric spectra is the use of empirical atomistic force fields. Instead, to study the contributions to the dielectric spectrum in atomic detail, the interaction potentials must be optimized to ensure that the water-water, the water-ion, and the ion-ion interactions are consistent with the experimentally determined thermodynamic properties. Therefore, to study the effects of ions on aqueous solutions in molecular detail, and in particular at finite concentrations, the development of reliable force fields is crucial. ${ }^{22,25-28}$

In this paper, we perform a direct comparison between simulated and experimental dielectric spectra of $\mathrm{Na}_{2} \mathrm{SO}_{4}$ and 
$\mathrm{MgSO}_{4}$ solutions at concentrations up to $2 \mathrm{M}$, using a newly optimized force field for $\mathrm{SO}_{4}^{2-}$. To guarantee the correct thermodynamic behavior, we have concurrently optimized the $\mathrm{SO}_{4}^{2-}$ force field with respect to the solvation free energy and the activity coefficient, ensuring an accurate representation of the water-ion and ion-ion interaction potentials. Dielectric spectra cover two different aspects of electrolyte solutions: (1) the dielectric constant, which is the zero-frequency limit of the dielectric response function, and (2) the relaxation dynamics. For $\mathrm{Na}_{2} \mathrm{SO}_{4}$, our simulations reproduce both the experimental concentration-dependent decrease in the static dielectric constant and the spectral shape (the real part decreasing monotonically with increasing frequency and the imaginary part consisting of a single peak) quantitatively. For $\mathrm{MgSO}_{4}$, a slow mode at a frequency much lower than the bulk water relaxation frequency is found, in agreement with experimental findings. Interpretation of these results is complex, especially since the addition of ions also drastically changes the water contribution to the dielectric spectrum. Therefore, we use spectral decomposition to unambiguously show that the slow mode in the simulations of $\mathrm{MgSO}_{4}$ solutions is due to ion-pair relaxation. Interestingly, this ionic dielectric contribution has a pronounced satellite peak in the $\mathrm{THz}$ regime, which corresponds well with recent $\mathrm{THz}$ absorption experiments on $\mathrm{MgSO}_{4}$ solutions. ${ }^{11}$ Whereas the $\mathrm{GHz}$ ion-pair contribution is caused by ion-pair rotation, we attribute the $\mathrm{THz}$ contribution to the vibration of water-separated ion pairs. By comparison with our previous spectral results for $\mathrm{NaCl}$ solutions, ${ }^{17,21}$ we conclude that whereas monovalent sodium-halide solutions show only negligible traces of ion-pair dielectric relaxation, the spectrum of divalent $\mathrm{MgSO}_{4}$ solutions is in fact dominated by ion-pair relaxation over the entire $\mathrm{GHz}$ range. Also for the mixed monovalent-divalent $\mathrm{Na}_{2} \mathrm{SO}_{4}$ solutions, the spectral decomposition indicates a significant ionic contribution, but the water contribution still dominates. Our results underline the dominant role of ion valency and show that multivalent ion relaxation can outweigh water solvation dynamics over the entire frequency range from $\mathrm{GHz}$ to $\mathrm{THz}$.

\section{METHODS}

\section{A. Molecular dynamics (MD) simulations}

The pair interaction potential $U_{i j}$ between atoms $i$ and $j$ is modeled through a sum of Coulomb and Lennard-Jones (LJ) interactions,

$$
U_{i j}\left(r_{i j}\right)=\frac{\lambda_{q} q_{i} q_{j}}{4 \pi \varepsilon_{0} r_{i j}}+\lambda_{\mathrm{LJ}} 4 \varepsilon_{i j}\left[\frac{\sigma_{i j}^{12}}{r_{i j}^{12}}-\frac{\sigma_{i j}^{6}}{r_{i j}^{6}}\right],
$$

where $q_{i}, q_{j}$ are the charges of the atoms $i$ and $j, r_{i j}$ is the distance between these atoms, $\sigma_{i j}$ is the effective radius, and $\varepsilon_{i j}$ is the interaction strength. We use $\lambda_{q}=\lambda_{\mathrm{LJ}}=1$ for all intermolecular interactions. The Lorentz-Berthelot combination rules are used for the Lennard-Jones parameters, scaled by a factor for anion-cation interactions,

$$
\varepsilon_{i j}=\sqrt{\varepsilon_{i} \varepsilon_{j}} \quad \text { and } \quad \sigma_{i j}=\lambda_{\sigma}\left(\sigma_{i}+\sigma_{j}\right) \frac{1}{2} .
$$

The factor $\lambda_{\sigma}$ is only allowed to differ from 1 for the anioncation interactions if necessary to reproduce both the solvation free energy and the activity coefficient.
We use a mechanically flexible model for $\mathrm{SO}_{4}^{2-}$, with the intramolecular interactions turned off $\left(\lambda_{q}=\lambda_{\mathrm{LJ}}=0\right)$, except for the $\mathrm{O}-\mathrm{O}$ interaction, where we use Coulomb interactions scaled by a prefactor $\lambda_{q}=0.833$ and $\lambda_{\mathrm{LJ}}=0$ (set by the FudgeQQ and FudgeLJ parameters in GROMACS). We employ the SPC/E water model,${ }^{32}$ which has a rigid geometry. The bond length of $1.0 \AA$ and bond angle of $109.47^{\circ}$ are fixed using the SHAKE algorithm. ${ }^{33}$ Intramolecular interactions are irrelevant for this rigid model.

All simulations are performed using the GROMACS 4.5.4 $4^{34,35}$ software. Periodic boundary conditions are applied in all three directions, and particle-mesh Ewald summation with a grid spacing of $0.12 \mathrm{~nm}$ in conjunction with tinfoil boundary conditions is used to handle long-ranged electrostatic forces. ${ }^{36}$ The simulations are carried out in the NVT (for equilibration) and NPT (for production) ensembles using Parrinello-Rahman coupling to keep the pressure at $1 \mathrm{bar}^{37}$ and a Nosé-Hoover thermostat to keep the temperature at $300 \mathrm{~K}$. The $\mathrm{LJ}$ interactions are truncated at $r_{c}=1.1 \mathrm{~nm}$ with a shift function for the range $1.0<r_{i j}<1.1 \mathrm{~nm}$ to make the potential approach zero smoothly. ${ }^{35}$

\section{B. Force field optimization}

We optimize the force field for $\mathrm{SO}_{4}^{2-}$ based on the scheme developed previously. ${ }^{27,28} \mathrm{Here}$, we give a brief overview of the method (see the Appendix for details). First, we calculate the isolines in the $\sigma-\varepsilon$ space of the sulfate oxygens along which the experimental sulfate solvation free energy is reproduced. Along the solvation free energy isolines, we calculate the activity derivative, and compare with the experimental value at a given concentration. Since the activity is not reproduced for any $\sigma-\varepsilon$ combination for $\mathrm{MgSO}_{4}$, we change the combination rule for the Lennard-Jones interaction between the magnesium and the sulfate oxygen via the scaling parameter $\lambda_{\sigma}$ in Eq. (2). The optimal parameters for sulfate are summarized in Table I, together with the rest of the force field parameters.

\section{Dielectric spectra}

The complex frequency-dependent dielectric susceptibility $\chi(f)=\chi^{\prime}(f)-i \chi^{\prime \prime}(f)$ relates the total system polarization $\vec{P}(f)$ to the electric field $\vec{E}(f)$ via the linear-response relation $\vec{P}(f)=V \epsilon_{0} \chi(f) \vec{E}(f)$, where $V$ is the system volume and $\epsilon_{0}$ is the vacuum permittivity. According to the fluctuation dissipation theorem, $\chi(f)$ follows from the autocorrelation of equilibrium polarization fluctuations via ${ }^{40,41}$

$$
\chi(f)=-\frac{1}{3 V k_{\mathrm{B}} T \epsilon_{0}} \int_{0}^{\infty} e^{-2 \pi i f t}\langle\vec{P}(0) \dot{\vec{P}}(t)\rangle \mathrm{d} t,
$$

where $\dot{\vec{P}}(t)$ denotes the time derivative of the time-dependent total polarization $\vec{P}(t)$ and $k_{\mathrm{B}} T$ is the thermal energy. Equation (3) allows us to obtain the complete dielectric spectrum from a single simulation trajectory in equilibrium.

The dielectric response of the electrolyte is decomposed into three terms,

$$
\Delta \chi(f)=\chi_{\mathrm{w}}(f)+\chi_{\mathrm{IW}}(f)+\Delta \chi_{\mathrm{I}}(f),
$$


TABLE I. Lennard-Jones parameters and atomic partial charges. Unless noted otherwise, Lorentz-Berthelot mixing rules $\left(\lambda_{\sigma}=1, \lambda_{\varepsilon}=1\right)$ are used for $\sigma_{i j}$ and $\varepsilon_{i j}$. The intramolecular 1-3 Coulomb interactions are taken into account within a single $\mathrm{SO}_{4}^{2-}$ ion, scaled by the factor $\lambda_{q}$. For all intermolecular interactions $\lambda_{q}$ $=\lambda_{\mathrm{LJ}}=1$.

\begin{tabular}{|c|c|c|c|c|}
\hline Site & $\sigma_{i}(\mathrm{~nm})$ & $\varepsilon_{i}(\mathrm{~kJ} / \mathrm{mol})$ & $q(|e|)$ & Ref. \\
\hline S & 0.355 & 1.0465 & 2 & Cannon-McCammon 29 \\
\hline $\mathrm{O}$ of $\mathrm{SO}_{4}$ & 0.3916 & 0.1 & -1 & This work \\
\hline $\mathrm{Mg}^{2+}$ & 0.263 & 0.004 & 2 & Mamatkulov-Fyta-Netz ${ }^{30}$ \\
\hline $\mathrm{Na}^{+}$ & 0.258 & 0.4186 & 1 & Smith-Dang ${ }^{31}$ \\
\hline $\mathrm{Cl}^{-}$ & 0.440 & 0.4186 & -1 & Smith-Dang ${ }^{31}$ \\
\hline $\mathrm{O}$ of water & 0.3166 & 0.65 & -0.8476 & $\mathrm{SPC} / \mathrm{E}^{32}$ \\
\hline $\mathrm{H}$ of water & 0.000 & 0.00 & 0.4238 & $\mathrm{SPC} / \mathrm{E}^{32}$ \\
\hline Bond & Interaction function & $\mathrm{r}_{0}(\mathrm{~nm})$ & $\mathrm{k}_{b}\left(\mathrm{~kJ} \mathrm{~mol}^{-1} \mathrm{~nm}^{-2}\right)$ & \\
\hline $\mathrm{S}-\mathrm{O}$ & $\left(\frac{1}{2}\right) k_{b}\left(r-r_{0}\right)^{2}$ & 0.15 & $3.7656 \times 10^{4}$ & This work \\
\hline Angle & Interaction function & $\theta_{0}$ (degrees) & $\mathrm{k}_{\theta}\left(\mathrm{kJ} \mathrm{mol}^{-1} \mathrm{rad}^{-2}\right)$ & \\
\hline $\mathrm{O}-\mathrm{S}-\mathrm{O}$ & $\left(\frac{1}{2}\right) k_{\theta}\left(\theta-\theta_{0}\right)^{2}$ & 109.5 & 520.0 & This work \\
\hline Interaction & $\lambda_{\mathrm{LJ}}$ & $\lambda_{q}$ & $\lambda_{\sigma}$ & \\
\hline $\mathrm{O}-\mathrm{O}$ of $\mathrm{SO}_{4}$ & 0.0 & 0.833 & & This work \\
\hline $\mathrm{S}-\mathrm{O}$ of $\mathrm{SO}_{4}$ & 0.0 & 0.0 & & This work \\
\hline $\mathrm{Mg}-\mathrm{SO}_{4}$ & & & 1.65 & This work \\
\hline
\end{tabular}

where $\chi_{\mathrm{W}}$ is the water contribution, $\chi_{\mathrm{IW}}$ the water-ion crosscontribution, and $\Delta_{\chi_{\mathrm{I}}}$ the contribution from ion pair relaxation. The $\Delta$ indicates that the direct current (DC) conductivity of the ions has been subtracted (see the supplementary material and our previous work ${ }^{17,21}$ for a detailed explanation).

To compute the dielectric spectra, we simulate three separate trajectories of 100-ns duration each for the salt types $\mathrm{Na}_{2} \mathrm{SO}_{4}$ and $\mathrm{MgSO}_{4}$. The simulation boxes contain about 6000 water molecules each, with 150 ion pairs for the $1.5 \mathrm{M}$ $\mathrm{Na}_{2} \mathrm{SO}_{4}$ solution and 240 ion pairs for the $2.2 \mathrm{M} \mathrm{MgSO}_{4}$ solution. Simulations are performed with a 2 -fs integration time step and trajectories are saved every $10 \mathrm{fs}$. Because of memory limitations, all trajectories are split into series of 20-ns length for data analysis. The polarization components of each time series are Fourier transformed via fast Fourier transformation. Correlation functions are calculated by multiplication in Fourier space according to the convolution theorem. After back transformation into the time domain, the correlation functions are averaged over the different time series. Upon calculating the dielectric response from the averaged correlation functions, an upper time cutoff is imposed on the Fourier transform [Eq. (3)], as is discussed in detail in the supplementary material.

By construction, the rigid SPC/E water model is not expected to reproduce the water dynamics at frequencies in the mid-infrared regime, where the intramolecular degrees of freedom dominate the dielectric spectrum. In fact, the rigid SPC/E model has been found to be accurate up to a frequency of around $6 \mathrm{THz}$, above which the simulations fail to reproduce the hydrogen bond stretch vibration peak ${ }^{42}$ (even though the libration peak at $15 \mathrm{THz}$ is typically reproduced well). Therefore, we concentrate our analysis on the $\mathrm{GHz}$ and low $\mathrm{THz}$ regime.

\section{RESULTS AND DISCUSSION}

\section{A. Dielectric spectra}

We show the simulated real and imaginary dielectric functions for $\mathrm{Na}_{2} \mathrm{SO}_{4}$ solutions at three different concentrations by solid lines in Figs. 1(a) and 1(b). For comparison, we also include the pure water spectra from our previous work, ${ }^{17}$ shown in red. The broken lines are fits according to the Cole-Cole function,

$$
\Delta \chi(f)+1=\frac{\epsilon_{\mathrm{CC}}}{1+(i 2 \pi f \tau)^{1-\alpha}}+\epsilon_{\infty}
$$

defining the static dielectric constant $\epsilon_{\mathrm{CC}}$, the dielectric relaxation time $\tau$, and the exponent $\alpha$ as free fit parameters. In our fits of the simulation data, for which we use a custom Python program, we fix $\epsilon_{\infty}=1$ since our force fields do not include high-frequency atomic polarization effects. We show the experimental spectra (symbols) in Figs. 1(c) and 1(d), together with triple-Debye fits (solid lines) as employed in the original work, ${ }^{38}$ obtained for $n=3$ from the expression

$$
\Delta \chi(f)+1=\sum_{k=1}^{n} \frac{\epsilon_{k}}{1+i 2 \pi f \tau_{k}}+\epsilon_{\infty} .
$$

The red circles denote the experimental data for pure water ${ }^{39}$ and the red solid line represents a single Debye fit $[n=1$ in Eq. (6)]. The fits in Figs. 1(e) and 1(f) demonstrate that the experimental data are equally well described by the simpler Cole-Cole function (broken lines), which involves only three fit parameters.

In Figs. 1(g)-1(i) we compare the Cole-Cole fit parameters from experiments (crosses) and simulations (triangles) for all concentrations. The fit parameter $\epsilon_{\mathrm{CC}}$ in Fig. 1(g), 


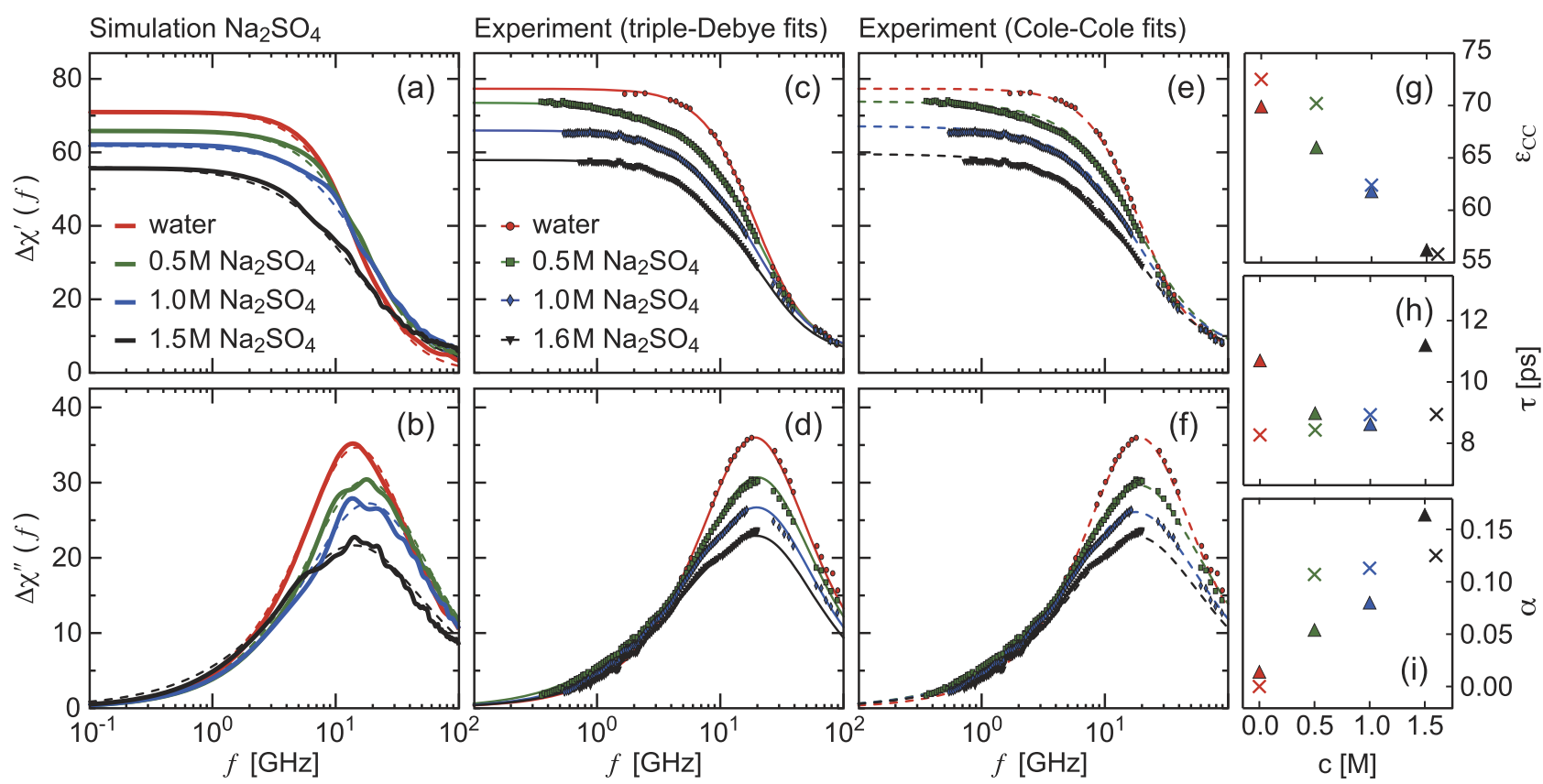

FIG. 1. Comparison of simulated and experimental dielectric spectra of $\mathrm{Na}_{2} \mathrm{SO}_{4}$ solutions at concentrations $c=0,0.5,1.0$, and $1.5 \mathrm{M}$ (in experiments $1.6 \mathrm{M}$ ). [(a) and (b)] Simulation data (solid lines) and Cole-Cole fits (broken lines) of the real and imaginary parts of the dielectric susceptibility $\Delta \chi^{\prime}(f)$ and $\Delta \chi^{\prime \prime}(f)$. [(c) and (d)] Experimental data (symbols) and triple-Debye fits (solid lines) of $\Delta \chi^{\prime}(f)$ and $\left.\Delta \chi^{\prime \prime}(f)\right)^{38}$ The red lines show single-Debye fits for pure water corresponding to $c=0 \mathrm{M}$. The experimental data are taken from Ref. 39. [(e) and (f)] The experimental data (symbols) with Cole-Cole fits (broken lines). [(g)-(i)] Direct comparison of the Cole-Cole fit parameters between experiments (crosses) and simulations (triangles).

which accounts for the microwave contribution to the static dielectric constant, shows a very good agreement between experiments and simulations. In particular, the strong decrease with rising salt concentration is accurately reproduced. The relaxation time $\tau$ in Fig. 1(h) increases only slightly with concentration in experiments, whereas we observe a nonmonotonic trend in our simulations. The deviation is presumably caused by a combination of sampling issues in the simulations, the uneven distribution of data points in the experimental spectra, and increasing calibration errors in the experimental data at low frequencies due to the strong rise of DC conductivity with increasing concentration. The parameter $\alpha$ in Fig. 1(i), which describes the peak shape, and thereby deviations from a simple Debye form, shows the same trend in simulations and experiments. Overall, the agreement between simulation and experiment is good.

In Fig. 2, we compare the dielectric spectra of $\mathrm{MgSO}_{4}$ solutions from experiments at different concentrations ${ }^{10}$ with

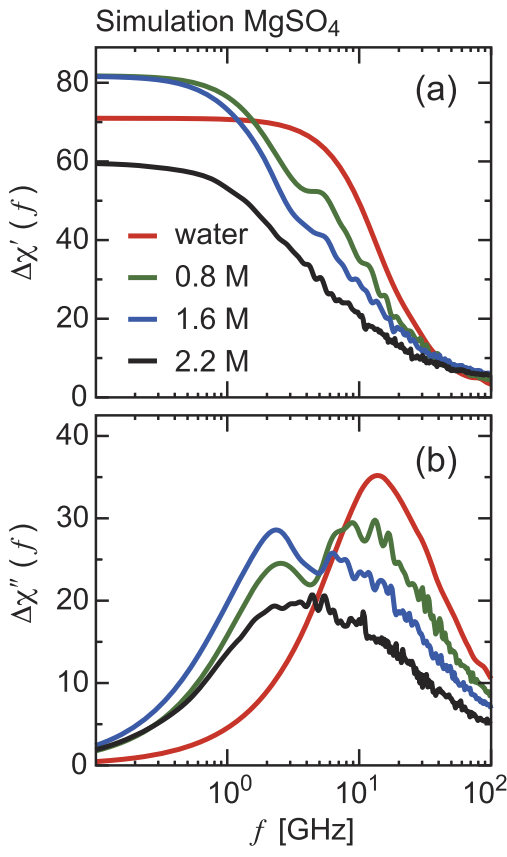

Experiment $\mathrm{MgSO}_{4}$
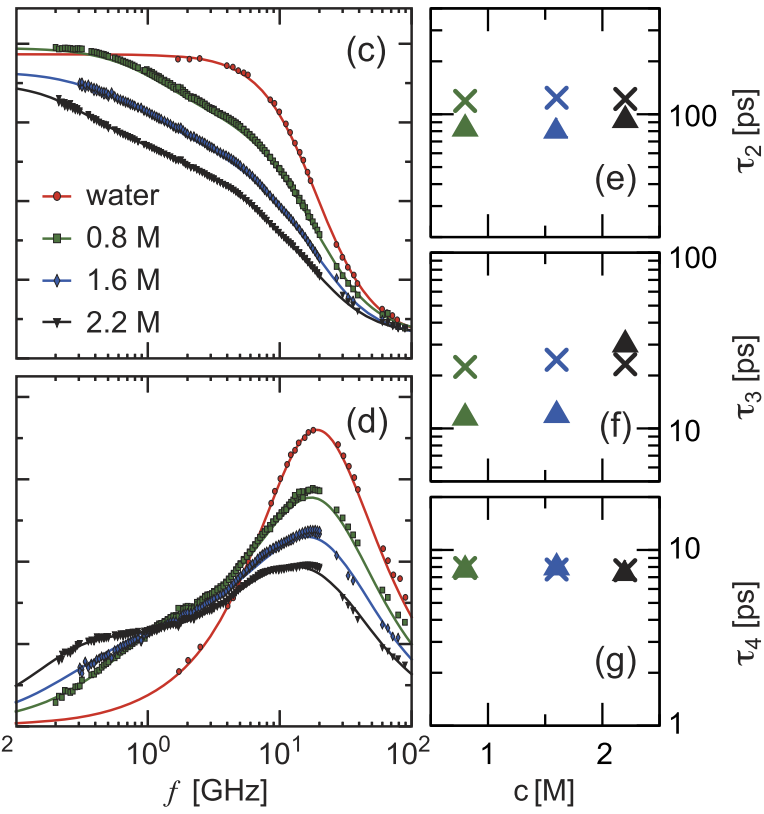

FIG. 2. Comparison of simulated and experimental dielectric spectra of $\mathrm{MgSO}_{4}$ solutions at matching concentrations $c=0,0.8,1.6$, and 2.2M. [(a) and (b)] Simulated real and imaginary parts of the dielectric susceptibility $\Delta \chi^{\prime}(f)$ and $\Delta \chi^{\prime \prime}(f)$. [(c) and (d)] Experimental data (symbols) and quadruple-Debye fits (solid lines) of $\Delta \chi^{\prime}(f)$ and $\Delta \chi^{\prime \prime}(f) .{ }^{10}$ The red lines show the results for pure water $(c=0 \mathrm{M})$. [(e)-(g)] The results for $\tau_{2}, \tau_{3}$, and $\tau_{4}$ of a quadruple Debye fit of the simulations [Eq. (6) with $n=4$, triangles] compared with the experimental results reported in Ref. 10 (crosses). 
simulation results. In Figs. 2(c) and 2(d), we show the experimental data (symbols) together with quadruple-Debye fits [ $n=4$ in Eq. (6), indicated by solid lines] as used in the original work. ${ }^{10}$ Similar to the experimental data, the simulated static dielectric constant passes through a maximum with increasing salt concentration, as can be read off from the low-frequency limit of the real part of the dielectric spectrum in Fig. 2(a). The gradual appearance of a slow process with rising salt concentration is clearly seen in the sub- $\mathrm{GHz}$ range of the experimental absorption spectra in Fig. 2(d). The interpretation of the simulated spectra in Figs. 2(a) and 2(b) is more difficult than for the $\mathrm{Na}_{2} \mathrm{SO}_{4}$ solutions because of the presence of slowly equilibrating ion-pairing processes. In particular, for $\mathrm{MgSO}_{4}$ solutions, the shape and the amplitude of the low-frequency dielectric contribution depends sensitively on the choice of the Fourier integration cutoff of the ionic auto-correlation function, as discussed in detail in the supplementary material. Clearly, however, a slow process appears in the simulated spectra with increasing salt concentration, shown in Fig. 2(b). We use a quadruple Debye fit to the simulation data to quantify the associated relaxation times and compare them with the experimental data. In the experimental spectra, peaks appear around $300 \mathrm{MHz}$ (associated with the slowest relaxation time $\tau_{1}$, attributed to ion pairs separated by two solvation shells) and $1.3 \mathrm{GHz}$ (the second-slowest relaxation time $\tau_{2}$, attributed to single-solvent-separated ion pairs). ${ }^{10}$ The slowest relaxation found in experiments, $\tau_{1}$, is not detectable in the simulations due to limitations on the simulation time. Therefore, the relaxation time of the slowest mode detected in the simulated spectra, centered around $1.9 \mathrm{GHz}$, equals $\tau_{2}$ $\approx 85 \mathrm{ps}$, which underestimates the experimental result $\left(\tau_{2}\right.$ $\approx 120$ ps) by about $30 \%$, see Fig. 2(e). The intermediate peak in the simulations, $12<\tau_{3}<30 \mathrm{ps}$, depending on the concentration, agrees reasonably well with the experimental values, $22<\tau_{3}<25$ ps [Fig. 2(f)]. The simulations reproduce the relaxation time of the water $\left(\tau_{4}=8 \mathrm{ps}\right)$ with high accuracy [Fig. 2(g)]. We conclude that the existence of a slow mode is clearly visible in our simulations in accordance with experimental results.

To understand the origin of the slow mode in the $\mathrm{MgSO}_{4}$ spectra, we decompose the dielectric spectra into the waterpair, the ion-pair, and the water-ion contributions according to Eq. (4). In Fig. 3 we compare the results for $1.5 \mathrm{M} \mathrm{Na}_{2} \mathrm{SO}_{4}$ and for $2.2 \mathrm{M} \mathrm{MgSO}_{4}$ with our previous results for $2.0 \mathrm{M} \mathrm{NaCl}$. The ion-water cross contribution (shown in green) only gives a small negative contribution for all solutions. Likewise, the ionic contribution (red solid line) is completely negligible for the monovalent salt $\mathrm{NaCl}$. In contrast, the ionic contribution is sizable for the spectra involving the divalent sulfate ion. In fact, for $\mathrm{MgSO}_{4}$, the ionic part dominates over the water contribution. Interestingly, the water contribution occurs in the 10-20 GHz range for all three salt solutions, and we see that the slow mode at $1-4 \mathrm{GHz}$, which is prominently visible in the $\mathrm{MgSO}_{4}$ spectrum, is entirely due to ion-pair dielectric relaxation effects. Therefore, the decomposition of the simulated spectra underpins the interpretation of this slow process in the experimental spectra as being caused by ion pairing. ${ }^{10}$ This is nontrivial, because we show in Fig. 3(f) that the drastic differences to the pure $\mathrm{H}_{2} \mathrm{O}$ spectrum upon addition of $\mathrm{NaCl}$ are solely caused by the salt-induced modification of the water contribution and cannot be explained by trivial dilution effects. ${ }^{17}$

At high frequency, the ionic contributions for $\mathrm{MgSO}_{4}$ and $\mathrm{Na}_{2} \mathrm{SO}_{4}$ [Figs. 3(b) and 3(d)] show a satellite peak in the $\mathrm{THz}$ range, indicative of a fast vibrational mode. This demonstrates that the ion-ion correlations produce dielectric signals in a very wide frequency range. However, the ion-ion polarization correlations in the $\mathrm{THz}$ range cannot make up for the
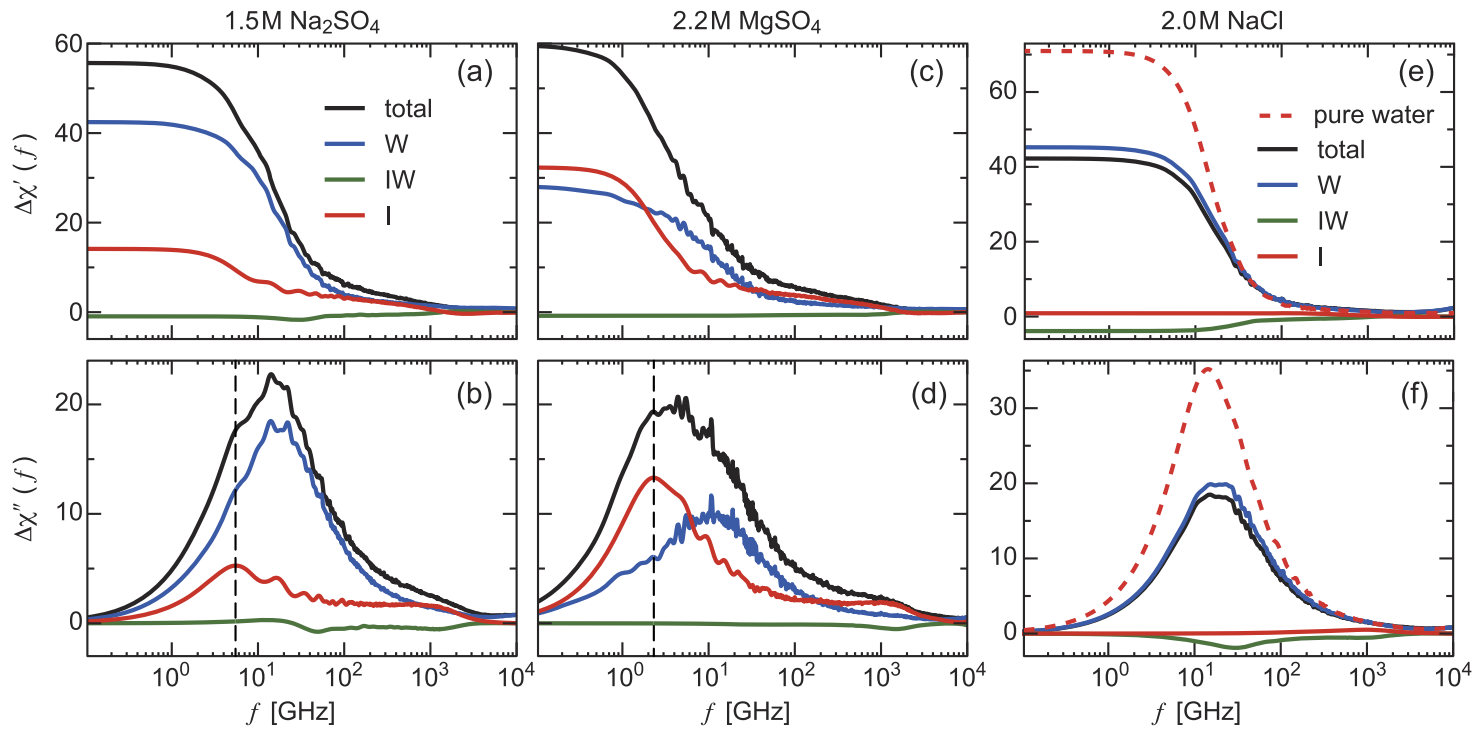

FIG. 3. Decomposition of the simulated real and imaginary parts of the dielectric spectra for [(a) and (b)] $1.5 \mathrm{M} \mathrm{Na}_{2} \mathrm{SO}_{4}$ and [(c) and (d)] $2.2 \mathrm{M} \mathrm{MgSO}$. In (e) and (f), we show the decomposition for $2.0 \mathrm{M} \mathrm{NaCl}$ taken from our previous work. ${ }^{21}$ For reference, we show the spectrum of pure $\mathrm{H}_{2} \mathrm{O}$ as a broken red line. The total $\Delta \chi(f)$ (black) is decomposed into water-pair (blue), ion-water (green), and ion-pair (red) contributions. The vertical broken lines at positions $f=5.3$ $\mathrm{GHz}$ for $\mathrm{Na}_{2} \mathrm{SO}_{4}$ (b) and $f=2.2 \mathrm{GHz}$ for $\mathrm{MgSO}_{4}$ (d) denote the maxima in the ion-pair contributions, which are explained by ion-pair rotational relaxation, as explained in the text. 
decrease in water-water polarization correlations, so that the overall dielectric adsorption above $10 \mathrm{GHz}$ still decreases with rising salt concentration [Figs. 1(b) and 2(b)]. Due to this dominance of the water-water contribution, a satellite peak is not expected to be detectable within the limited frequency range of the experiments. ${ }^{11}$ However, the presence of ion-ion correlations at high frequency provides a possible reason for the experimental observation that $\mathrm{MgSO}_{4}$ shows a large effect on the hydration water dynamics, whereas $\mathrm{Mg}^{2+}$ and $\mathrm{SO}_{4}^{2-}$ individually (in combination with other ions, such as $\mathrm{ClO}_{4}^{-}$, and $\mathrm{Cs}^{2+}$ ) do not. ${ }^{11}$

To elucidate the origin of the differences in the ionic spectral contribution between the different salt types, we show the radial distribution functions $g(r)$ of the ions in Fig. 4. Whereas the cations and anions in $\mathrm{Na}_{2} \mathrm{SO}_{4}$ solutions form contact pairs, as can be seen from the almost matching peak positions of the $g(r)$ of $\mathrm{SO}_{4}^{2-}-\mathrm{Na}^{+}$and $\mathrm{SO}_{4}^{2-}-\mathrm{H}_{2} \mathrm{O}$ [broken vertical lines in Figs. 4(a) and 4(c)], the dominant ion pairs in $\mathrm{MgSO}_{4}$ solutions are separated by a single layer of solvent molecules, as is evident from the shifted peak positions of the $g(r)$ of $\mathrm{SO}_{4}^{2-}$ $\mathrm{Mg}^{2+}$ and $\mathrm{SO}_{4}^{2-}-\mathrm{H}_{2} \mathrm{O}$ [broken vertical lines in Figs. 4(b) and 4(d)]. The differences between the ionic contributions to the spectra of $\mathrm{Na}_{2} \mathrm{SO}_{4}$ and $\mathrm{MgSO}_{4}$ solutions can be rationalized by analyzing the positions of the first peak in the cation-anion $g(r)$, which are found to be $0.38 \mathrm{~nm}$ for $\mathrm{Na}_{2} \mathrm{SO}_{4}$ and 0.50 $\mathrm{nm}$ for $\mathrm{MgSO}_{4}$ [broken vertical lines in Figs. 4(a) and 4(b)].

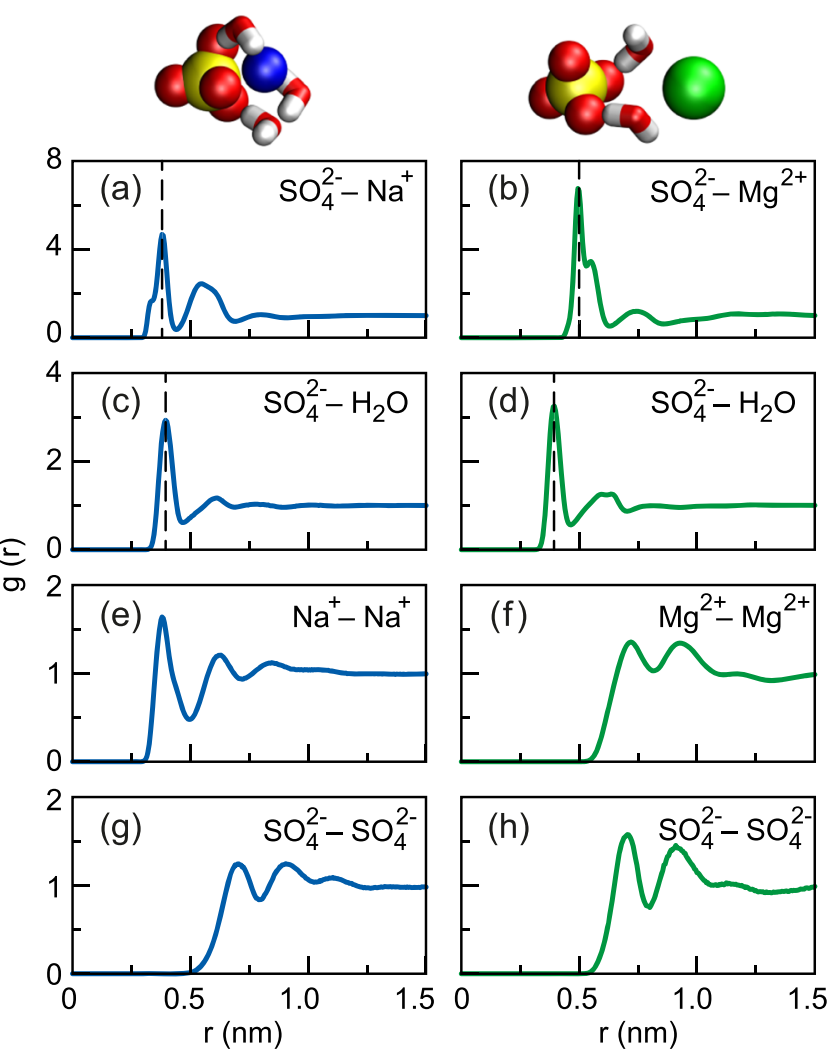

FIG. 4. Radial distribution functions of the ions in $1.5 \mathrm{M} \mathrm{Na}_{2} \mathrm{SO}_{4}$ (left) and 2.0 $\mathrm{M} \mathrm{MgSO}_{4}$ (right) solutions. The position of the sulfur has been used as the position of $\mathrm{SO}_{4}^{2-}$ and the position of the oxygen as the position of $\mathrm{H}_{2} \mathrm{O}$. The broken lines in (a)-(d) denote the maxima, which are used to estimate the typical separation of ion pairs. We find direct contact pairs for $\mathrm{Na}^{+}-\mathrm{SO}_{4}^{2-}$ and water-separated ion pairs for $\mathrm{Mg}^{2+}-\mathrm{SO}_{4}^{2-}$. The snapshots correspond to the maxima in (a)-(d).
Based on the scaling of the rotational relaxation time of a rigid rod with the third power of its length, ${ }^{43}$ the decrease in the relaxation frequency of $\mathrm{MgSO}_{4}$ relative to $\mathrm{Na}_{2} \mathrm{SO}_{4}$ ion pairs is estimated to be a factor $(0.50 / 0.38)^{3}=2.3$. In the simulations, the ion-pair relaxation frequencies, defined as the peak positions of the ion-pair spectral contributions [broken vertical lines in Figs. 3(b) and 3(d)], are equal to $2.2 \mathrm{GHz}$ for $\mathrm{MgSO}_{4}$ and $5.3 \mathrm{GHz}$ for $\mathrm{Na}_{2} \mathrm{SO}_{4}$, differing by a factor $5.3 / 2.2=2.4$. The good agreement suggests that rotational relaxation of nearest-neighbor ion pairs dominates the low-frequency ionic spectrum. These results could not be confirmed at lower ion concentrations, owing to numerical sampling issues of the ionic spectral contributions at lower concentrations. The high amplitude of the ionic relaxation peak of $\mathrm{MgSO}_{4}$ [Fig. 3(d)] relative to $\mathrm{Na}_{2} \mathrm{SO}_{4}$ [Fig. 3 (b)], which depends on the squared dipole moment of the ion pairs, is also consistent with the higher valency and the larger ion-ion distance of $\mathrm{MgSO}_{4}$ (see the supplementary material). This analysis shows that the frequency and amplitude of the slow ionic relaxation peak, and therefore its discernibility, are determined by the ion valencies and the ion-ion radial distribution functions. In contrast, we rationalize the high-frequency contribution, which is visible in the $\mathrm{THz}$ range, by ionic vibration of nearest-neighbor ion pairs. As shown by the amplitude and sharpness of the first peak in the anion-cation $g(r)$ of $\mathrm{Na}_{2} \mathrm{SO}_{4}$ and $\mathrm{MgSO}_{4}$ solutions, these ion pairs are numerous and tightly bound. We estimate the vibration frequency of the ion-pairs as $f_{v i b}=\frac{1}{2 \pi} \sqrt{k / \mu}$, with $\mu$ being the reduced mass of the ion pair and $k$ being the effective spring constant. For each anion-cation pair, we estimate $k$ by fitting a quadratic function to the first well of the free energy $F(r)=-k_{\mathrm{B}} T \ln g(r)$ (see the supplementary material). For both $\mathrm{MgSO}_{4}$ and $\mathrm{Na}_{2} \mathrm{SO}_{4}$, the estimated upper bound of the vibration frequency equals $f_{v i b} \approx 2 \mathrm{THz}$, which is consistent with the appearance of the high-frequency satellite peaks in the ion-pair contributions in Figs. 3(b) and 3(d). In the supplementary material, we show that the high-frequency satellite peak is also present at lower salt concentrations.

\section{CONCLUSIONS}

Using extensive equilibrium molecular dynamics simulations based on a new thermodynamically optimized $\mathrm{SO}_{4}^{2-}$ force field, we extract the dielectric spectra of $\mathrm{Na}_{2} \mathrm{SO}_{4}$ and $\mathrm{MgSO}_{4}$ solutions at different concentrations and compare directly with experimental data. The overall agreement is good, which shows that classical force fields are able to describe ion-specific shifts of dielectric constants, dielectric relaxation times, and even the shape of the dielectric spectra very well. By a decomposition of the dielectric spectra of $\mathrm{NaCl}, \mathrm{Na}_{2} \mathrm{SO}_{4}$, and $\mathrm{MgSO}_{4}$ solutions into the contributions from water-water, ion-ion, and water-ion correlations, we show that ion-pairing contributions are only detectable when the divalent $\mathrm{SO}_{4}^{2-}$ ions are present in the solution. For $\mathrm{MgSO}_{4}$ solutions, the ionic contribution in fact dominates over the water contribution. Interestingly, the ion-pair dielectric contribution for the $\mathrm{SO}_{4}^{2-}$ salts exhibits an extremely broad spectrum, encompassing slow processes in the sub-GHz range, but also fast processes in the $\mathrm{THz}$ range. Whereas the slow processes can be traced back to slow rotation of ion pairs, the fast processes are rationalized 
by vibrations of the interionic distance. Since we find only solvent-separated ion pairs in $\mathrm{MgSO}_{4}$ solutions, these vibrations therefore involve sandwiched and strongly correlated water molecules, confirming the experimental observation that an unexpectedly high number of water molecules are involved in the $\mathrm{THz}$ relaxation of $\mathrm{MgSO}_{4}$ solutions. ${ }^{11}$ As we show in the supplementary material, the sandwiched water molecules in solvent-separated ion pairs give rise to pronounced signals in the self-part of the dielectric water spectrum. In conclusion, our results point to a delicate and hitherto neglected coupling between ion and water dielectric relaxation phenomena for electrolyte solutions containing divalent ions, with wideranging implications for the interpretation of microwave and $\mathrm{THz}$ spectroscopy experiments.

\section{SUPPLEMENTARY MATERIAL}

See supplementary material for details of the procedure used to obtain the autocorrelation functions, the spectral analysis of solutions at different concentrations, and the free energy of ion-pair interactions.

\section{ACKNOWLEDGMENTS}

S.I.M. acknowledges financial support from the Marie Curie International Incoming Fellowship (No. call FP7PEOPLE-2009-IIF) and the State S\&T program of the Republic of Uzbekistan (No. BF2-027). R.R.N. acknowledges support from the DFG as part of the SFB 1078 "Protonation Dynamics in Protein Function." We gratefully acknowledge computing time on the HPC cluster at ZEDAT, Freie Universität Berlin.

\section{APPENDIX: SULFATE FORCE FIELD OPTIMIZATION DETAILS}

In this appendix, we describe the development of a force field for the $\mathrm{SO}_{4}^{2-}$ ion in SPC/E water, by a systematic doubleoptimization strategy based on the experimental ion solvation free energy and the experimental activity coefficients. Whereas the ion solvation free energy is a single-ion property that describes the binding of solvation water to the ion, the activity coefficient is a sensitive measure of ion pairing properties at finite concentration and has been used previously to successfully parameterize ionic force fields. ${ }^{26-28,30,44}$ Only such a double optimization can unambiguously determine the two Lennard Jones (LJ) force-field parameters corresponding to the $\mathrm{LJ}$ radius $\sigma$ and the $\mathrm{LJ}$ interaction strength $\varepsilon$. The free parameters available for the optimization of the sulfate ion are the LJ parameters of the oxygen and the sulfur, the latter of which we disregard because the sulfur is hidden inside the sulfate. Furthermore, because of its polyatomic nature, there is the molecular flexibility of the $\mathrm{SO}_{4}^{2-}$ ion and the partial charges on its atoms. Finally, while the LJ parameters of the sulfate-water, sodium-water, and magnesium-water interactions are fixed by the solvation free energy optimization, the sulfate-counterion interactions can be set independently. Usually, these interactions are inferred from the ion-water interaction parameters using a combination rule, but these rules are purely heuristic. Therefore, as a final optimization parameter, we consider a modification factor of the standard combination rule for sulfate-counterion interactions. Our optimization proceeds in two steps. First, we optimize the LJ parameters of the sulfate oxygen atoms to reproduce the solvation free energy of a single sulfate ion in water, while keeping the sulfur atom LJ parameters fixed. This yields a subset of optimal LJ parameters, which we refer to as the solvation free energy isoline. In the second step, we compute the activity derivatives of aqueous sodium and magnesium sulfate solutions for the $\mathrm{LJ}$ parameters on the solvation free energy isoline. Our $\mathrm{SO}_{4}^{2-}$ model has no atomic polarizability; however, some molecular polarizability in the form of flexible bonds and angles is necessary to reproduce the experimental activity. We find that a rigid model of $\mathrm{SO}_{4}^{2-}$ underestimates the activity, whereas the solvation free energy is largely insensitive to the flexibility of the molecule. This result stands in clear contrast to recent results for $\mathrm{H}_{3} \mathrm{O}^{+}$and $\mathrm{OH}^{-},{ }^{28}$ where both the solvation free energy and the activity can be captured using a rigid model. For $\mathrm{Na}_{2} \mathrm{SO}_{4}$, a LJ parameter combination exists, which simultaneously matches the experimental ion solvation free energy and the experimental activity coefficient. For $\mathrm{MgSO}_{4}$, by contrast, the simulated activity derivative significantly differs from the experimental value for all $\mathrm{LJ}$ parameter combinations that lie on the optimized solvation free energy isoline. We also study the relevance of the partial charges of the sulfate ion on the $\mathrm{MgSO}_{4}$ solution activity derivatives but find no effect, from which we conclude that the partial charge distribution is not a crucial optimization parameter. Consequently, for magnesium sulfate solutions we adjust the combination rule for the sulfate-magnesium $\mathrm{LJ}$ radius to match the experimental activity derivative at a single concentration. Finally, we verify that the calculated activity derivatives for $\mathrm{Na}_{2} \mathrm{SO}_{4}$ and $\mathrm{MgSO}_{4}$ solutions using our optimal force fields match the experimental activity for concentrations up to $2.0 \mathrm{M}$ and thus validate our new thermodynamically consistent force field for sulfate ions.

\section{Sulfate-magnesium interactions and the flexibility of sulfate}

The sulfate ion consists of a central sulfate atom surrounded by four oxygen atoms in tetrahedral arrangement. The equilibrium geometry of $\mathrm{SO}_{4}^{2-}$ in water has an $\mathrm{O}-\mathrm{S}-\mathrm{O}$ angle of $109.5^{\circ}$ and $\mathrm{S}-\mathrm{O}$ bond length of $0.15 \mathrm{~nm} .{ }^{29}$ Because of the high partial charges on the constituent atoms, however, this geometry is sensitive to the presence of water and other ions, which is important for the activity of sulfate solutions. We have performed $a b$ initio computations to check how the sulfate geometry responds to the presence of a magnesium ion. The potential energy surface scan of $\mathrm{MgSO}_{4}$ in the gas phase (vacuum) and in water employing a polarizable continuum model (PCM) is performed at the MP2/6311++G(p,d) level. All calculations are carried out with the GAUSSIAN 09 program package. ${ }^{45}$

Potential energy curves as a function of the distance between $\mathrm{Mg}^{2+}$ and $\mathrm{SO}_{4}^{2-}$ in the gas phase and in the watermimicking dielectric environment are shown in Fig. 5(a). In the gas phase the equilibrium distance between the $\mathrm{S}$ and $\mathrm{Mg}$ nuclei is $R_{\min }=0.250 \mathrm{~nm}$, whereas in water this distance increases to $R_{\min }=0.275 \mathrm{~nm}$. Geometry optimization 


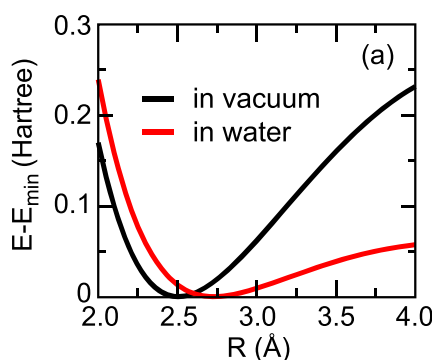

(d)

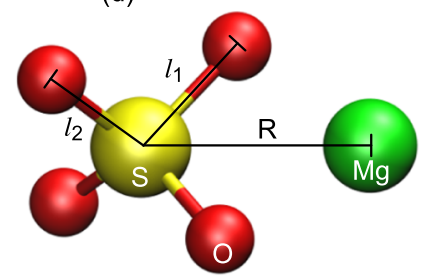

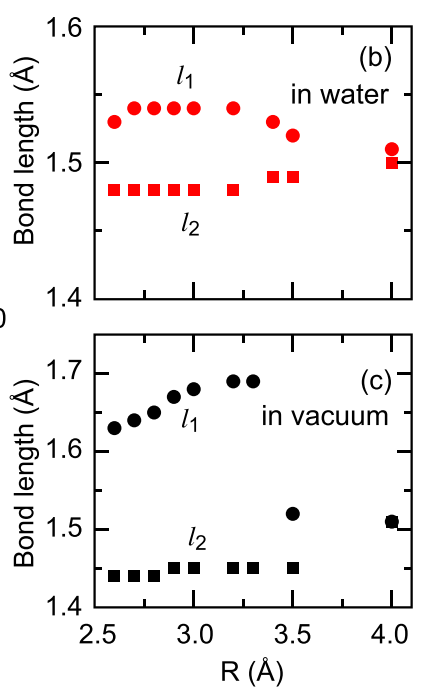

FIG. 5. (a) Potential energy scan for $\mathrm{MgSO}_{4}$ computed at the MP2/6311++G(p,d) level. In vacuum the equilibrium distance between $S$ and $\mathrm{Mg}$ is $\mathrm{R}_{\min }(\mathrm{S}-\mathrm{Mg})=0.25 \mathrm{~nm}$. In water (using a polarizable continuum model), this distance shifts to $0.275 \mathrm{~nm}$. Calculated intramolecular bond lengths of $\mathrm{SO}_{4}^{2-}$ depend on the $\mathrm{S}-\mathrm{Mg}$ distance in (b) vacuum and (c) water. Both in vacuum and water the $\mathrm{S}-\mathrm{O}$ bond lengths facing $\mathrm{Mg}^{2+}$ are elongated while those facing away are shortened. (d) Picture of the $\mathrm{Mg}-\mathrm{SO}_{4}$ system where the distance $R$, one of the two bond lengths facing $\mathrm{Mg}^{2+}\left(l_{1}\right)$, and one of the two bond lengths facing away $\left(l_{2}\right)$ are indicated.

shows that the approach of $\mathrm{Mg}^{2+}$ significantly distorts the sulfate ion in vacuum as well as in water, as reflected by the distance-dependent changes of the two distinct $\mathrm{SO}$ bond lengths in Figs. 5(b) and 5(c). These results show that $\mathrm{SO}_{4}^{2-}$ is flexible and responds to the presence of a nearby $\mathrm{Mg}^{2+}$ ion, which has to be taken into account in classical MD simulations for a correct description of sulfate properties in solution. In fact, it turns out that the commonly used rigid models ${ }^{29}$ for $\mathrm{SO}_{4}^{2-}$ significantly overestimate the degree of association in aqueous solution, which leads to excessive contact ion pairing and unrealistic clustering ${ }^{46}$ which we also verify in our present work. One part of the solution to this problem is to make the bonds and angles flexible, governed by finite bond length and bond angle interaction potentials, while at the same time including scaled intramolecular Coulomb interactions within $\mathrm{SO}_{4}^{2-}$ to maintain a stable tetrahedral shape. Accordingly, in this work, we include intramolecular 1-3 electrostatic interactions between the oxygen atoms in the sulfate ion (whereas by default non-bonded interactions between 1 and 3 neighboring atoms are excluded in GROMACS), scaled by a fudge factor of $\lambda_{q}=0.833$, and thereby enhance the rigidity of the $\mathrm{O}-\mathrm{S}-\mathrm{O}$ bond angle.

\section{Solvation free energy}

To calculate the single-ion solvation free energy, a sulfate ion is placed in a cubic box of size $L=2.5 \mathrm{~nm}$ containing $506 \mathrm{SPC} / \mathrm{E}$ water molecules. A cut-off distance of $r_{c}=1.0 \mathrm{~nm}$ is chosen for the Lennard-Jones interactions, and long-range corrections for energy and pressure due to dispersion interactions are used in all simulations. MD simulations are performed with a time-step of $1 \mathrm{fs}$.

The solvation free energy of the sulfate ion is calculated using the integral ${ }^{48}$

$$
\begin{aligned}
\Delta G_{\text {sim }}= & \int_{0}^{1}\left\langle\frac{\partial H_{\phi}\left(\phi_{L J}, \phi_{C}=0\right)}{\partial \phi_{L J}}\right\rangle d \phi_{L J} \\
& +\int_{0}^{1}\left\langle\frac{\partial H_{\phi}\left(\phi_{L J}=1, \phi_{C}\right)}{\partial \phi_{C}}\right\rangle d \phi_{C},
\end{aligned}
$$

where $H_{\phi}$ is the Hamiltonian of the system, $\phi_{L J}$ and $\phi_{C}$ are the $\mathrm{LJ}$ and charge transition coordinates which are 0 in the initial state and 1 in the final state. The solvation path is split in two separate processes: in the first step a neutral van der Waals particle is created, which is assigned a charge in the second step. Along the transition path, the $\phi$-dependent Hamiltonian is defined as

$$
H_{\phi}\left(\phi_{L J}, \phi_{C}\right)=H\left\{\begin{array}{l}
q_{\phi}=\phi_{C} q \\
\sigma_{\phi}=\left[1-\left(1-\phi_{L J}\right)^{k}\right] \sigma \\
\varepsilon_{\phi}=\left[1-\left(1-\phi_{L J}\right)^{k}\right] \varepsilon
\end{array}\right\},
$$

with $q, \sigma$, and $\varepsilon$ being the partial charge, the LJ radius, and the LJ interaction strength, respectively, in the state $\phi_{L J}$ $=\phi_{C}=1$, and $H$ being the unperturbed Hamiltonian. We set the exponent $k$ in Eq. (A2) equal to $k=6$ to avoid divergences. To reduce the computational effort, we exclude the intra-molecular interactions from the integration pathway and verify the accuracy of that procedure by comparing the result to the energy difference between $\Delta G_{\text {sim }}$ calculated in vacuum and $\Delta G_{\text {sim }}$ calculated in bulk water, both with full intramolecular electrostatics included in the integration pathway. The difference between the approaches with and without intramolecular interactions included-which is not a priori zero because the flexibility of the molecule could lead to different distributions of molecular conformations in the vacuum and the water-amounts to only $3 \mathrm{~kJ} / \mathrm{mol}$, which falls within the range of accuracy of our optimization. Integrations are performed through a 12-point Gaussian quadrature with $\phi$ $\in\{0.00922,0.04794,0.11505,0.20634,0.31608,0.43738$, $0.56262,0.68392,0.79366,0.88495,0.95206,0.99078\}$. For every value of $\phi$, we perform a $250 \mathrm{ps}$ simulation of which the first $50 \mathrm{ps}$ are discarded for equilibration.

The ionic solvation free energy computed in the simulations is sensitive to the simulation scheme (system shape, periodic or finite system) and treatment of the electrostatic forces. Therefore, for comparison with experimental data, several corrections have to be applied to the simulation data. The correction term accounting for finite system and ion size reads ${ }^{49}$

$$
\Delta G_{f s}=\frac{z^{2} e^{2}}{4 \pi \varepsilon_{0}}\left[\left(1+\frac{1}{\varepsilon_{r}}\right)\left(\frac{2 \pi R_{i o n}^{2}}{3 L^{3}}-\frac{4 \pi^{2} R_{i o n}^{5}}{45 L^{6}}\right)-\frac{\xi_{\text {ew }}}{2 \varepsilon_{r}}\right],
$$

where $z$ is the ion valency and $e$ the elementary charge. Here $R_{i o n}$ is the effective radius of the ion which is estimated from the first peak of the ion-oxygen radial distribution function, and $\varepsilon_{r}=71$ is the relative dielectric constant of SPC/E water. The Wigner potential equals $\xi_{\text {ew }}=-2.837279 / L$, where $L$ denotes the simulation box size in $\mathrm{nm}$.

Experimental values of the solvation free energy are usually given with respect to a hypothetical transfer of ions from the ideal gas phase at $p_{0}=1 \mathrm{~atm}$ pressure to the ideal solution at a pressure of $p_{1}=24.6 \mathrm{~atm}$, corresponding to a density of $1 \mathrm{~mol} / \mathrm{l}$. Thus, it is also necessary to include a correction term 
related to the compression of the gas,

$$
\Delta G_{\text {press }}=k_{B} T \ln \left(p_{1} / p_{0}\right)=7.9 \mathrm{~kJ} / \mathrm{mol},
$$

where $k_{B} T$ is the thermal energy.

Hence, the total single-ion solvation free energy is given by

$$
\Delta G_{\text {solv }}=\Delta G_{\text {sim }}+\Delta G_{f s}+\Delta G_{\text {press }} .
$$

Experimental solvation free energy data are available only for neutral ion-pairs, for which the water surface potential drops out. Extracting single-ion solvation free energies from experimental data therefore relies on a reference value, for which traditionally the solvation free energy of the proton $\Delta G_{\text {solv }}\left(\mathrm{H}^{+}\right)$has been used. However, the most popular estimates for $\Delta G_{s o l v}\left(\mathrm{H}^{+}\right)$, those of Tissandier et al. ${ }^{50}(-1104.5$ $\mathrm{kJ} / \mathrm{mol})$ and Marcus $^{47}(-1056 \mathrm{~kJ} / \mathrm{mol})$, differ by about 50 $\mathrm{kJ} / \mathrm{mol}$. Therefore, we choose the chloride ion instead, with the commonly used Smith-Dang parameters ${ }^{31}$ given in Table I. Taking Smith-Dang parameters for the $\mathrm{Cl}^{-}$ion and SPC/E water, $\sigma_{\mathrm{Cl}-\mathrm{O}_{W}}=0.378 \mathrm{~nm}$ and $\varepsilon_{\mathrm{Cl}-\mathrm{O}_{W}}=0.52 \mathrm{~kJ} / \mathrm{mol}$, and using the data from Ref. 51, MD simulations yield a $\mathrm{Cl}^{-}$solvation free energy value of $\Delta G_{\text {solv }}=-306 \mathrm{~kJ} / \mathrm{mol}$. For $\mathrm{SO}_{4}^{2-}$, the reference solvation free energy is the difference between the experimental sulfate and chloride solvation free energies. Using the experimental values for $\mathrm{SO}_{4}^{2-}$ and $\mathrm{Cl}^{-}$taken from Ref. 47 we find

$$
\begin{aligned}
\Delta \Delta G & =\Delta G_{\text {solv }}\left(\mathrm{SO}_{4}^{2-}\right)-z \times \Delta G_{\text {solv }}\left(\mathrm{Cl}^{-}\right) \\
& =-1090-2 \cdot(-347)=-396 \mathrm{~kJ} / \mathrm{mol},
\end{aligned}
$$

where $z=2$ is the valency of the sulfate anion. For $\mathrm{Na}^{+}$, we use Smith-Dang parameters ${ }^{31}$ and for $\mathrm{Mg}^{2+}$ we take our previously optimized force field. ${ }^{30}$

Using the procedure explained above, we first compute the solvation free energy of $\mathrm{SO}_{4}^{2-}$ using the original rigid force field proposed by Cannon et al. ${ }^{29}$ Using the set of LJ parameters for $\mathrm{SO}_{4}^{2-}$ reproduced in Table I, we obtain $\Delta G_{\text {sim }}=-970$ $\mathrm{kJ} / \mathrm{mol}$ for the free energy of charging the rigid molecule from $q=0$ to $q=-2 e$ which is in fair agreement with the value of $\Delta G_{\text {sim }}=-945 \mathrm{~kJ} / \mathrm{mol}$ obtained in the work of Cannon et al. ${ }^{29}$ The difference between our work and Ref. 29 might be due to different cutoff settings, as well as to differences in system size and correction terms. We find that the solvation free energy of $\mathrm{SO}_{4}^{2-}$ is rather insensitive to variations of the $\mathrm{LJ}$ parameters of the sulfur atom (data not shown) and mainly depends on parameters of the sulfate oxygen atoms. Therefore we look for the combinations of $\sigma_{\mathrm{O}}^{\mathrm{SO}_{4}}$ and $\varepsilon_{\mathrm{O}}^{\mathrm{SO}_{4}}$ that most closely reproduce the experimental solvation free energy of $\mathrm{SO}_{4}^{2-}$.

In Fig. 6(a) we plot the solvation free energy difference of the flexible sulfate ion and two Smith-Dang $\mathrm{Cl}^{-}$ions, $\Delta \Delta G$, as a function of $\sigma_{\mathrm{O}}^{\mathrm{SO}_{4}}$ for a few fixed value of $\varepsilon_{\mathrm{O}}^{\mathrm{SO}_{4}}$. For all curves the sulfur atom LJ parameters are set to $\sigma_{S}=0.355$ $\mathrm{nm}$ and $\varepsilon_{S}=1.0465 \mathrm{~kJ} / \mathrm{mol},{ }^{29}$ angle and bond parameters of the sulfate ion are shown in Table I. For increasing values of $\sigma_{\mathrm{O}}^{\mathrm{SO}_{4}}$ and $\varepsilon_{\mathrm{O}}^{\mathrm{SO}_{4}}$ the solvation process becomes less favorable and the solvation free energy increases, as shown in Fig. 6(a). The horizontal line shows the experimental value $\Delta \Delta G=-396$ $\mathrm{kJ} / \mathrm{mol}$; we see that for each value of the LJ interaction strength $\varepsilon_{\mathrm{O}}^{\mathrm{SO}_{4}}$ there is a value of the $\mathrm{LJ}$ radius $\sigma_{\mathrm{O}}^{\mathrm{SO}_{4}}$ that reproduces the experimental solvation free energy. The resulting combination of $\mathrm{LJ}$ parameters that reproduce the experimental value


FIG. 6. (a) Solvation free energy difference $\Delta \Delta G$ between a flexible sulfate ion and two chloride ions as a function of the sulfate oxygen LJ radius $\sigma_{\mathrm{O}}^{\mathrm{SO}_{4}}$. The red horizontal line represents the experimental value $\Delta \Delta G=-396$ $\mathrm{kJ} / \mathrm{mol}^{47}$ Symbols represent simulation results at different values of $\varepsilon_{\mathrm{O}}^{\mathrm{SO}_{4}}$. The sulfur atom LJ parameters are fixed at $\sigma_{S}=0.355 \mathrm{~nm}$ and $\varepsilon_{S}=1.0465$ $\mathrm{kJ} / \mathrm{mol}$ and the partial charges are fixed at $q_{S}=2 e$ and $q_{O}=-1 e$. (b) The combinations of $\sigma_{\mathrm{O}}^{\mathrm{SO}_{4}}$ and $\varepsilon_{\mathrm{O}}^{\mathrm{SO}_{4}}$ that reproduce the experimental solvation free energy difference of the sulfate and reference chloride ion as obtained from (a). The circles show the LJ parameters used in simulations to calculate the activity coefficient of $\mathrm{Na}_{2} \mathrm{SO}_{4}$ and $\mathrm{MgSO}_{4}$ solutions. The optimal force field corresponds to $\sigma_{\mathrm{O}}^{\mathrm{SO}_{4}}=0.391 \mathrm{~nm}$ and $\varepsilon_{\mathrm{O}}^{\mathrm{SO}_{4}}=0.1 \mathrm{~kJ} / \mathrm{mol}$ (filled green circle). The LJ parameters of two literature force fields are denoted as a blue triangle ${ }^{46}$ and a red square ${ }^{29}$ for comparison.

of $\Delta \Delta G$ is shown in Fig. 6(b) as a continuous line. The $\mathrm{LJ}$ parameters used to compute activity coefficients of $\mathrm{Na}_{2} \mathrm{SO}_{4}$ and $\mathrm{MgSO}_{4}$ are denoted by circles. For comparison, previous $\mathrm{LJ}$ force field parameters are indicated by a red square ${ }^{29}$ and by a blue triangle. ${ }^{46}$

Note that the free energy of a rigid $\mathrm{SO}_{4}^{2-}$ ion with $\mathrm{LJ}$ parameters on the isoline of the flexible ion shown in Fig. 6(b) differs less than $2 \mathrm{~kJ} / \mathrm{mol}$ from the free energy of the flexible ion, as we have determined in separate simulations, the results of which are not shown.

\section{Solution activity}

The Kirkwood-Buff (KB) theory connects integrals over pair distribution functions, the so-called KB integrals, to thermodynamic quantities such as isothermal compressibilities, partial molar volumes, and, in particular, solution activity derivatives. ${ }^{44}$ The KB integrals are defined as

$$
G_{i j}^{\infty}=4 \pi \int_{0}^{\infty}\left(g_{i j}-1\right) r^{2} \mathrm{~d} r,
$$

where $g_{i j}$ is the radial distribution function between species $i$ and $j$. To calculate the activity, we need the following integrals over the salt $(s)$, cations $(+)$, anions $(-)$, and water $(w)$ radial distribution functions

$$
\begin{aligned}
G_{s s}^{\infty}= & {\left[\frac{\rho_{+}}{\rho_{+}+\rho_{-}}\right]^{2} G_{++}^{\infty}+\left[\frac{\rho_{-}}{\rho_{+}+\rho_{-}}\right]^{2} G_{--}^{\infty} } \\
& +\left[\frac{\rho_{+} \rho_{-}}{\left(\rho_{+}+\rho_{-}\right)^{2}}\right]\left(G_{+-}^{\infty}+G_{-+}^{\infty}\right), \\
G_{s w}^{\infty}= & {\left[\frac{\rho_{+}}{\rho_{+}+\rho_{-}}\right] G_{+w}^{\infty}+\left[\frac{\rho_{-}}{\rho_{+}+\rho_{-}}\right] G_{-w}^{\infty} }
\end{aligned}
$$

where $\rho_{+}$and $\rho_{-}$are the concentrations of cations and anions in solution. The electroneutrality condition can be expressed as $G_{+w}^{\infty}=G_{-w}^{\infty}$ and $^{52,53}$

$$
G_{+-}^{\infty}=\frac{1}{\rho_{+}}+G_{++}^{\infty}=\frac{1}{\rho_{-}}+G_{--}^{\infty} .
$$


Using these KB integrals, the derivative of the salt activity, $a_{s s}$, follows as

$$
a_{s s}=1+\left.\left(\frac{\partial \ln y_{s}}{\partial \ln \rho_{s}}\right)\right|_{p, T}=\frac{1}{1+\left(\rho_{-}+\rho_{+}\right)\left(G_{s s}^{\infty}-G_{s w}^{\infty}\right)},
$$

where the salt activity coefficient $y_{s}$ is defined via $a_{s}=\rho_{s} y_{s}, \rho_{s}$ is the salt concentration, and $a_{s}$ is the salt activity. Additional details on the $\mathrm{KB}$ theory can be found elsewhere. ${ }^{44,53}$

The KB integrals defined in Eq. (A6) need to be truncated at a finite integration limit $R$. In infinite space, the two-particle radial distribution functions (RDFs) $g_{i j}(r)$, which are defined as the atomic density at a distance $r$ from a particular atom normalized on the average atomic density, equal to unity for $r \rightarrow \infty$. In a finite simulation box, however, RDFs $g_{i j}^{s i m}$ calculated from molecular dynamics simulations never reach 1 exactly because one particle is singled out and the remaining fluid is asymmetric. Although the deviation of $g_{i j}^{\text {sim }}(r \rightarrow \infty)$ from 1 is small, the spatial integral of Eq. (A6) diverges. To remedy this problem, we exploit the known cutoff dependence of the KB integral when the integral is truncated at $r=R$. The asymmetry of Eq. (A6) is taken into account by using a geometrical weight function $w(r, R)$, which differs from $4 \pi r^{2}$ for finite $R,{ }^{54}$

$$
G_{i j}^{R}(R)=\int_{0}^{R} w(r, R)\left(g_{i j}^{s i m}(r)-1\right) \mathrm{d} r,
$$

with

$$
w(r, R)=4 \pi r^{2}\left[1-\frac{3 r}{2 R}+\frac{r^{3}}{2 R^{3}}\right] .
$$

The difference between $G_{i j}^{\infty}$ and $G_{i j}^{R}(R)$ in Eq. (A10) scales linearly with $1 / R$, which allows us to extrapolate Eq. (A10) to infinite box size.$^{54}$ We have verified that the heuristic normalization of the $g_{i j}(r)$, as employed previously, ${ }^{30}$ gives very similar results.

The radial distribution functions needed for the calculation of activities are obtained using a cubic box containing 6966 SPC/E water molecules. MD simulations are performed with a 2-fs time step. To gather sufficient statistics, the particle trajectories are stored every $0.2 \mathrm{ps}$ and the total simulation time is $100 \mathrm{~ns}$. According to our two-step optimization procedure, we take combinations of $\mathrm{LJ}$ parameters that reproduce the experimental solvation free energy of the sulfate ion, shown in Fig. 6(b), and determine the activity derivatives for $\mathrm{Na}_{2} \mathrm{SO}_{4}$ and $\mathrm{MgSO}_{4}$ solutions at finite concentration.

Using the Smith-Dang LJ parameter for sodium, ${ }^{31}$ we calculate the activity derivatives of $\mathrm{Na}_{2} \mathrm{SO}_{4}$ at $1 \mathrm{M}$ as a function of the $\mathrm{LJ}$ radius $\sigma_{\mathrm{O}}^{\mathrm{SO}_{4}}$ in Fig. 7(a). The LJ parameters correspond to the circles that lie on the solvation free energy isoline shown in Fig. 6(b). The experimental data for the activities as a function of salt molality are obtained from Ref. 55 and the corresponding derivative of the activity with respect to molar concentration $a_{s s}$ is determined using Eq. (A9) and shown as a horizontal red line. It is seen that the activity derivative for $\mathrm{Na}_{2} \mathrm{SO}_{4}$ matches the experimental value at $\sigma_{\mathrm{O}}^{\mathrm{SO}_{4}}=3.916 \AA$ and $\varepsilon_{\mathrm{O}}^{\mathrm{SO}_{4}}=0.1 \mathrm{~kJ} / \mathrm{mol}$. This force field thus reproduces both the sulfate solvation free energy and the activity derivative of $\mathrm{Na}_{2} \mathrm{SO}_{4}$ at $1 \mathrm{M}$ and is denoted in Fig. 6(b) by a solid green circle. Note that the literature sulfate force field denoted by a
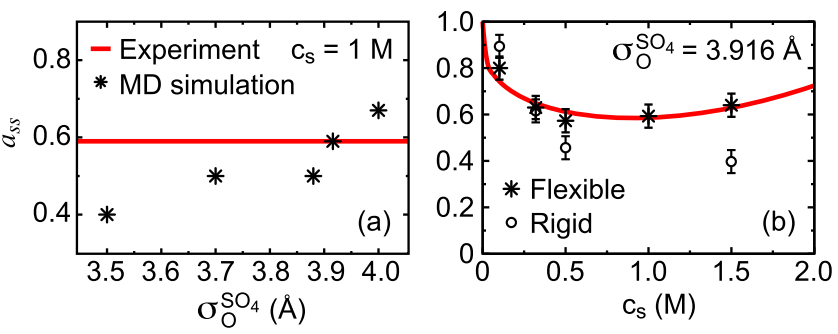

FIG. 7. (a) The activity coefficient derivative $a_{s s}$ of $\mathrm{Na}_{2} \mathrm{SO}_{4}$ at $1.0 \mathrm{M}$ as a function of $\sigma_{\mathrm{O}} \mathrm{SO}_{4}$ on the experimental solvation free energy isoline shown in Fig. 6(b). The experimental value is denoted by a horizontal line. (b) $a_{s s}$ as a function of salt concentration for the optimized LJ parameters and partial charges shown in Table I. The curve denotes the experimental activity derivative obtained from $y_{s}\left(\rho_{s}\right)$ using Eq. (A9). Also shown is $a_{s s}$ for a rigid molecule with the same LJ parameters as the flexible model.

red square in Fig. 6(b) has a much smaller LJ radius, ${ }^{29}$ and our results for the activity derivative $a_{s s}$ in Fig. 7(a) show that for small $\mathrm{LJ}$ radii $a_{s s}$ is significantly smaller than the experimental value, indicative of pronounced ion clustering in solution. ${ }^{27}$ In Fig. 7(b) the simulated activity derivative $a_{s s}$ of $\mathrm{Na}_{2} \mathrm{SO}_{4}$ is compared to the experimental curve as a function of salt concentration. It turns out that the optimized set of LJ parameters reproduces the experimental data (red line) for $a_{s s}$ over the entire concentration range. We also show $a_{s s}$ of a rigid model (open spheres) with the same LJ parameters as the flexible model, which has a solvation free energy within $2 \mathrm{~kJ} / \mathrm{mol}$ from the solvation free energy of the flexible ion. The behavior of $a_{s s}$ of the rigid ion as a function of concentration does not agree with experiment. The low values of $a_{s s}$ indicate too strong ion pairing, similar to the results from the rigid $\mathrm{SO}_{4}^{2-}$ force field of Ref. 29.

To understand the solution behavior in more detail, we show the radial distribution functions $g(r)$ for $\mathrm{Na}_{2} \mathrm{SO}_{4}$ at $0.5 \mathrm{M}$ in Fig. 8(a) using our double-optimized force field. We find that $\mathrm{Na}_{2} \mathrm{SO}_{4}$ preferably forms contact ion pairs [Fig. 8(a)]. The sulfate-sulfate radial distribution function displays a weak solvent-separated peak around $7 \AA$ [Fig. 8(b)] and the sodiumsodium radial distribution function displays a peak around


FIG. 8. The radial distribution functions of our optimized $\mathrm{NaSO}_{4}$ force field at $0.5 \mathrm{M}$ for (a) $\mathrm{SO}_{4}^{2-}-\mathrm{Na}^{+}$, (b) $\mathrm{SO}_{4}^{2-}-\mathrm{SO}_{4}^{2-}$, (c) $\mathrm{Na}^{+}-\mathrm{Na}^{+}$, and (d) $\mathrm{SO}_{4}^{2-}-\mathrm{H}_{2} \mathrm{O}$. In (d), the boundaries of the first two solvation shells are indicated by broken vertical lines, and the cumulative number of water molecules within a distance $r$ from the sulfate, $n(r)=n_{\text {bulk }} \int_{0}^{r} g(r) 4 \pi r^{2} d r$, is shown as a dotted line (right-hand axis). 

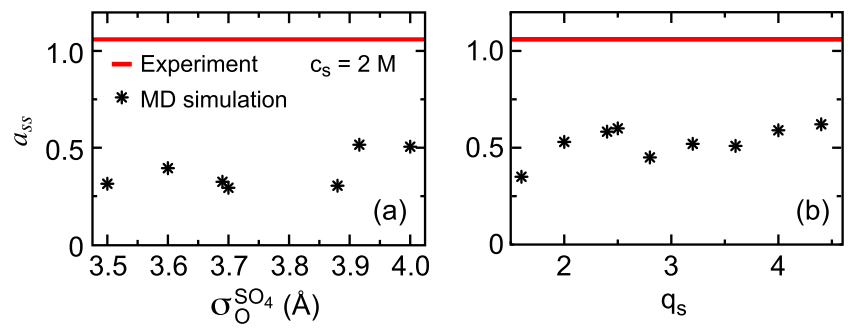

FIG. 9. (a) The activity coefficient derivative $a_{s s}$ of $\mathrm{MgSO}_{4}$ at $2.0 \mathrm{M}$ as a function of $\sigma_{\mathrm{O}}^{\mathrm{SO}_{4}}$ on the experimental solvation free energy isoline shown in Fig. 6(b). The experimental value, denoted by a horizontal line, is never reached. (b) $a_{s s}$ of $\mathrm{MgSO}_{4}$ at $2 \mathrm{M}$ as a function of the sulfur atom partial charge $q_{S}$ for the optimized LJ force field parameters. Note that $q_{S}=2$ is the standard value. ${ }^{29}$

3.5 Å due to the simultaneous pairing of more than one sodium ion to a single sulfate ion [Fig. 8(c)]. The sulfate-water $g(r)$ is shown in Fig. 8(d), with the boundaries of the first two solvation shells indicated with broken lines. The cumulative number of water molecules within a distance $r$ from the sulfate, $n(r)$, is shown as a dotted line. Interestingly, the sulfate ion is quite strongly hydrated with about 14 water molecules in the first solvation shell and about 30 water molecules in the second solvation shell [Fig. 8(d), dotted lines, right-hand scale], in good agreement with experimental data based on fs-resolved IR vibrational spectroscopy. ${ }^{11}$

In contrast to $\mathrm{Na}_{2} \mathrm{SO}_{4}$, for $\mathrm{MgSO}_{4}$ the simulated values of the activity derivative differ significantly from the experimental value at $2 \mathrm{M}$, as shown in Fig. 9(a). For $\mathrm{Mg}^{2+}$, we have used our recently derived parameters for $\mathrm{Mg}^{2+} .{ }^{30}$ Because with $\sigma_{\mathrm{O}}^{\mathrm{SO}_{4}}$ and $\varepsilon_{\mathrm{O}}^{\mathrm{SO}_{4}}$ alone the activity of $\mathrm{MgSO}_{4}$ solutions cannot be reproduced, we investigate the influence of the partial charge on the sulfur atom. We show $a_{s s}$ as a function of the sulfur atom partial charge $q_{S}$ for fixed Lennard-Jones parameters in Fig. 9(b). Note that the standard value used previously and in all our work is $q_{S}=2$. It is seen that the partial charge distribution in the sulfate ion has only a minor influence on the activity and therefore cannot be used as an optimization parameter.

\section{Ion-ion combination rule}

From the data in Fig. 9, we conclude that there is no combination of LJ parameters and partial charges that simultaneously reproduces experimental solvation free energies and activity coefficients for $\mathrm{MgSO}_{4}$ solutions. This is similar to our previous findings for magnesium halide solutions. ${ }^{30}$ Accordingly, we optimize the scaling factor $\lambda_{\sigma}$ which we have introduced in the cation-anion effective radius in Eq. (2), like we did previously. ${ }^{27,28,30}$ In Fig. 10(a) we present the activity derivative $a_{s s}$ for $\mathrm{MgSO}_{4}$ at $2.0 \mathrm{M}$ as a function of $\lambda_{\sigma}$. The symbols denote the simulated values and the black solid line is a fourth-order polynomial fit. The sulfate LJ parameters are the ones optimized for $\mathrm{Na}_{2} \mathrm{SO}_{4}$, and the cation-cation, anion-anion, and water-ion mixing rules are not modified. The experimental value for $a_{s s}$ is denoted by a horizontal red line. ${ }^{55} \mathrm{~A}$ perfect match between simulated and experimental activity derivatives is obtained for $\lambda_{\sigma}=1.65$, a value that is very similar to our previous results for $\mathrm{MgCl}_{2}, \mathrm{MgBr}_{2}$, and $\mathrm{MgI}_{2}$ solutions, ${ }^{30}$ which suggests that this scaling factor is more a signature of
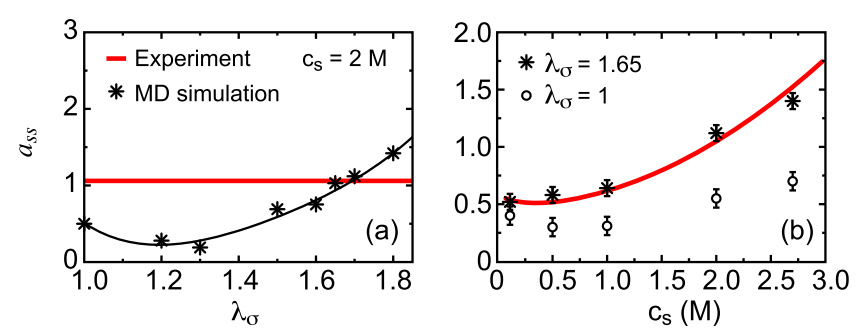

FIG. 10. (a) Activity derivative $a_{s s}$ of $\mathrm{MgSO}_{4}$ at $2 \mathrm{M}$ as function of the LJ radius scaling factor $\lambda_{\sigma}$. The symbols show simulation results, the black curve is a fourth-order polynomial fit meant as a guide to the eye. The horizontal red line denotes the experimental value. (b) $a_{s s}$ of $\mathrm{MgSO}_{4}$ as a function of concentration. The red curve shows the experimental data, the open symbols show simulation data for the unmodified combination rule $\lambda_{\sigma}=1$, and the black stars represent the simulation data for the optimized LJ radius scaling factor $\lambda_{\sigma}=1.65$.

the small divalent cation $\mathrm{Mg}^{2+}$ than of the sulfate anion. In Fig. 10(b) we present the simulated activity derivative using a fixed value $\lambda_{\sigma}=1.65$ for $\mathrm{MgSO}_{4}$ at different concentrations $c_{s}$ (star symbols). Clearly, this force field reproduces the experimental data (red solid line) over the entire concentration range (note that the experimental solubility limit of $\mathrm{MgSO}_{4}$ is reached at about $3 \mathrm{M}$ ). For comparison, we also include data for the optimized sulfate LJ parameters using the unmodified combination rule $\lambda_{\sigma}=1$ (open circles). The data fall significantly below the experimental values, indicative of excessive ion pairing, clustering, and underestimated solubility.

In Fig. 11 we compare the ionic radial distribution functions for $\mathrm{MgSO}_{4}$ at $2 \mathrm{M}$ for the (a) literature sulfate $\mathrm{LJ}$ parameters $^{29}$ and (b) the optimized sulfate LJ parameters for both the unmodified and the modified combination rule $\lambda_{\sigma}=1$ and $\lambda_{\sigma}=1.65$. Whereas pronounced contact ion-pairing is seen for all RDFs with the literature parameters in (a), the newly optimized LJ parameters in (b) (broken lines) eliminate contact ion pairing for the $\mathrm{Mg}^{2+}-\mathrm{Mg}^{2+}$ and $\mathrm{SO}_{4}^{2-}-\mathrm{SO}_{4}^{2-} \mathrm{RDFs}$ and shift the solvent-separated peaks to larger ion separations. Note that with the unmodified scaling factor $\lambda_{\sigma}=1$, denoted by the green solid line in (b), the $\mathrm{Mg}^{2+}-\mathrm{SO}_{4}^{2-} \mathrm{RDF}$ indicates
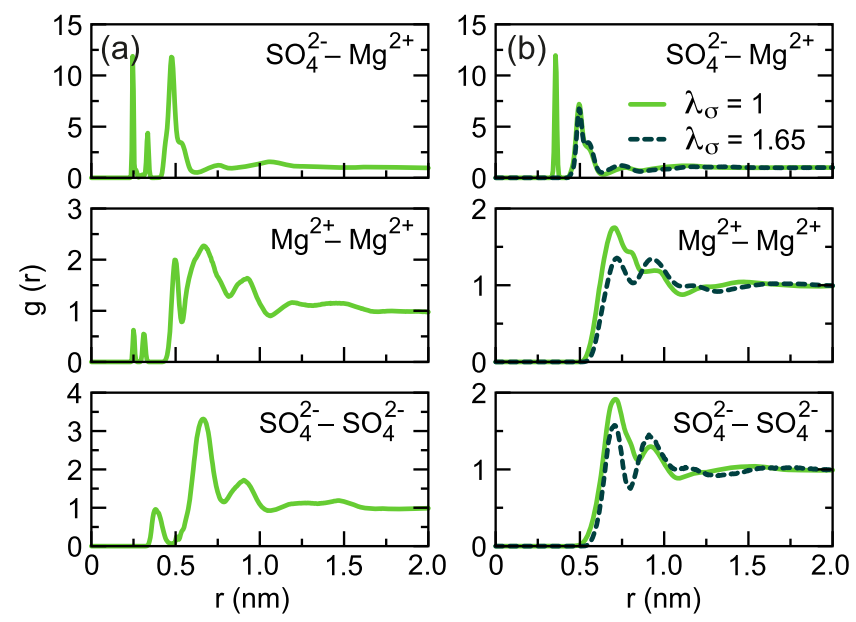

FIG. 11. Radial distribution functions of a $\mathrm{MgSO}_{4}$ solution at $2 \mathrm{M}$ using the force field proposed by Cannon et al. ${ }^{29}$ (a) and using our optimized force field given in Table I (b). The literature sulfate model shows pronounced magnesium-sulfate ion pairing. For our model with $\lambda_{\sigma}=1.65$ the magnesiumsulfate contact ion pair peak disappears and the distributions shift to larger distances, which explains the increase in $a_{s s}$ as $\lambda_{\sigma}$ grows. 
pronounced contact ion pairing. Only the increase from $\lambda_{\sigma}$ $=1$ to $\lambda_{\sigma}=1.65$, denoted by the broken line in (b), completely eliminates $\mathrm{Mg}^{2+}-\mathrm{SO}_{4}^{2-}$ contact pairs, while the $\mathrm{Mg}^{2+}-\mathrm{Mg}^{2+}$ and $\mathrm{SO}_{4}^{2-}-\mathrm{SO}_{4}^{2-} \mathrm{RDFs}$ are only slightly affected.

${ }^{1}$ D. Markovich, Physiol. Rev. 81, 1499 (2001).

${ }^{2}$ R. Langford, E. Hurrion, and P. A. Dawson, J. Genet. Genomics 44, 7 (2017).

${ }^{3}$ F. Hofmeister, Arch. Exp. Path. Pharm. 24, 247 (1888).

${ }^{4}$ P. Wingfield, Current Protocols in Protein Science Supplement 13, A.3F.1 (1998).

${ }^{5}$ Y. Marcus, Chem. Rev. 109, 1346 (2009).

${ }^{6}$ B. Hess and N. A. F. van der Vegt, Proc. Natl. Acad. Sci. U. S. A. 106, 13296 (2009).

${ }^{7}$ J. O'Brien, J. Prell, M. Bush, and E. Williams, J. Am. Chem. Soc. 132, 8248 (2010).

${ }^{8}$ K. Tielrooij, S. van der Post, M. Hunger, M. Bonn, and H. Bakker, J. Chem. Phys. B 115, 12638 (2011).

${ }^{9}$ G. Stirnemann, E. Wernersson, P. Jungwirth, and D. Laage, J. Am. Chem. Soc. 135, 11824 (2013).

${ }^{10}$ R. Buchner, T. Chen, and G. Hefter, J. Phys. Chem. B 108, 2365 (2004).

${ }^{11}$ K. J. Tielrooij, N. Garcia-Araez, M. Bonn, and H. J. Bakker, Science 328, 1006 (2010).

12 A. Y. Zasetsky and I. M. Svishchev, J. Chem. Phys. 115, 1448 (2001).

${ }^{13}$ A. Knocks and H. Weingärtner, J. Phys. Chem. B 105, 3635 (2001).

${ }^{14} \mathrm{C}$. Schröder, C. Wakai, H. Weingärtner, and O. Steinhauser, J. Chem. Phys. 126, 084511 (2007).

${ }^{15}$ J. Sala, E. Guàrdia, and J. Martí, J. Chem. Phys. 132, 214505 (2010).

${ }^{16}$ C. Schröder and O. Steinhauser, J. Chem. Phys. 132, 244109 (2010).

${ }^{17}$ K. Rinne, S. Gekle, and R. R. Netz, J. Phys. Chem. A 118, 11667 (2014).

${ }^{18}$ J. Barthel and R. Buchner, Chem. Soc. Rev. 21, 263 (1992).

${ }^{19}$ R. Buchner, G. T. Hefter, and P. M. May, J. Phys. Chem. A 103, 1 (1999).

${ }^{20} \mathrm{H}$. Weingärtner, A. Knocks, S. Boresch, P. Höchtl, and O. Steinhauser, J. Chem. Phys. 115, 1463 (2001).

${ }^{21}$ K. Rinne, S. Gekle, and R. Netz, J. Chem. Phys. 141, 214502 (2014).

${ }^{22}$ M. Sega, S. S. Kantorovich, C. Holm, and A. Arnold, J. Chem. Phys. 140, 211101 (2014).

${ }^{23}$ K. S. Cole and R. H. Cole, J. Chem. Phys. 9, 341 (1941).

${ }^{24}$ S. Havriliak and S. Negami, Polymer 8, 161 (1967).

${ }^{25}$ C. Oostenbrink, A. Villa, A. Mark, and W. van Gunsteren, J. Chem. Theory Comput. 25, 1656 (2004).

${ }^{26}$ M. Gee et al., J. Chem. Theory Comput. 7, 1369 (2011).

${ }^{27}$ M. Fyta and R. R. Netz, J. Chem. Phys. 136, 124103 (2012).

${ }^{28}$ D. J. Bonthuis, S. I. Mamatkulov, and R. R. Netz, J. Chem. Phys. 144, 104503 (2016)

${ }^{29}$ W. R. Cannon, B. M. Pettitt, and J. A. McCammon, J. Phys. Chem. 98, 6225 (1994).

${ }^{30}$ S. I. Mamatkulov, M. Fyta, and R. R. Netz, J. Chem. Phys. 138, 024505 (2013).
${ }^{31}$ L. X. Dang and D. E. Smith, J. Chem. Phys. 99, 6950 (1993).

${ }^{32}$ H. Berendsen, J. Grigera, and T. Straatsma, J. Phys. Chem. 91, 6269 (1987).

${ }^{33}$ J. P. Ryckaert, G. Ciccotti, and H. J. C. Berendsen, J. Comput. Phys. 23, 327 (1977).

${ }^{34}$ H. J. C. Berendsen, D. van der Spoel, and R. van Drunen, Comput. Phys. Commun. 91, 43 (1995).

${ }^{35}$ E. Lindahl, B. Hess, and D. van der Spoel, J. Mol. Model. 7, 306 (2001).

${ }^{36}$ T. Darden, D. York, and L. Pedersen, J. Chem. Phys. 98, 10089 (1993).

${ }^{37}$ M. Sprik, J. Hutter, and M. Parrinello, J. Chem. Phys. 105, 1142 (1996).

${ }^{38}$ R. Buchner, S. G. Capewell, G. Hefter, and P. M. May, J. Phys. Chem. B 103, 1185 (1999).

${ }^{39}$ J. Barthel, K. Bachhuber, R. Buchner, and H. Hetzenauer, Chem. Phys. Lett. 165, 369 (1990).

${ }^{40}$ S. R. de Groot and P. Mazur, Non-Equilibrium Thermodynamics (Dover, New York, 1984), pp. 150-156.

${ }^{41}$ J. M. Caillol, D. Levesque, and J. J. Weis, J. Chem. Phys. 85, 6645 (1986).

${ }^{42}$ A. Y. Zasetsky and V. I. Gaiduk, J. Phys. Chem. A 111, 5599 (2007).

${ }^{43}$ J. G. Kirkwood and P. L. Auer, J. Chem. Phys. 19, 281 (1951).

${ }^{44}$ S. Weerasinghe and P. Smith, J. Chem. Phys. 119, 11342 (2003).

${ }^{45}$ M. Frisch, G. W. Trucks, H. B. Schlegel, G. E. Scuseria, M. A. Robb, J. R. Cheeseman, G. Scalmani, V. Barone, B. Mennucci, G. A. Petersson, H. Nakatsuji, M. Caricato, X. Li, H. P. Hratchian, A. F. Izmaylov, J. Bloino, G. Zheng, J. L. Sonnenberg, M. Hada, M. Ehara, K. Toyota, R. Fukuda, J. Hasegawa, M. Ishida, T. Nakajima, Y. Honda, O. Kitao, H. Nakai, T. Vreven, J. A. Montgomery, Jr., J. E. Peralta, F. Ogliaro, M. Bearpark, J. J. Heyd, E. Brothers, K. N. Kudin, V. N. Staroverov, R. Kobayashi, J. Normand, K. Raghavachari, A. Rendell, J. C. Burant, S. S. Iyengar, J. Tomasi, M. Cossi, N. Rega, J. M. Millam, M. Klene, J. E. Knox, J. B. Cross, V. Bakken, C. Adamo, J. Jaramillo, R. Gomperts, R. E. Stratmann, O. Yazyev, A. J. Austin, R. Cammi, C. Pomelli, J. W. Ochterski, R. L. Martin, K. Morokuma, V. G. Zakrzewski, G. A. Voth, P. Salvador, J. J. Dannenberg, S. Dapprich, A. D. Daniels, Ö. Farkas, J. B. Foresman, J. V. Ortiz, J. Cioslowski, and D. J. Fox, Gaussian 09, Revision D.01, Gaussian, Inc., Wallingford, CT, 2009.

${ }^{46}$ E. Wernersson and P. Jungwirth, J. Chem. Theory Comput. 6, 3233 (2010).

${ }^{47}$ Y. Marcus, Ion Properties (Marcel Dekker, Inc., New York, 1997).

${ }^{48}$ T. P. Straatsma and J. A. Mccammon, Annu. Rev. Phys. Chem. 43, 407 (1992).

${ }^{49}$ C. Zhan and D. Dixon, J. Phys. Chem. A 106, 10311 (2002).

${ }^{50}$ M. D. Tissandier, K. A. Cowen et al., J. Chem. Phys. 102, 7787 (1998).

${ }^{51}$ D. Horinek, S. I. Mamatkulov, and R. R. Netz, J. Chem. Phys. 130, 124507 (2009).

${ }^{52}$ P. Kusalik and G. Patey, J. Chem. Phys. 86, 5110 (1987).

${ }^{53}$ Y. Ben-Naim and A. Marcus, J. Chem. Phys. 81(4), 2016 (1984).

${ }^{54}$ P. Krüger, S. Schnell, D. Bedeaux, S. Kjelstrup, T. J. H. Vlugt, and J. Simon, J. Phys. Chem. 4, 235 (2013).

${ }^{55}$ R. Robinson and R. Stokes, Electrolyte Solutions, 2nd ed. (Dover, New York, 2002). 